\title{
Orphanage at the FDA
}

\author{
Eric Chen
}

Received: 2 November 2010 / Accepted: 5 November 2010 /Published online: 16 November 2010

(C) Springer Science+Business Media, LLC 2010

In the late 1970s and early 1980s, social and political forces gave birth to a new movement to serve the needs of people with rare diseases, a movement that has only accelerated with the penetrance of internet connectivity. The FDA's Office of Orphan Products Development (OOPD) was established in 1982 to promote the development of products that demonstrate promise for the diagnosis, prevention, and/or treatment of rare diseases or conditions. Additional governmental responses included passage of the 1983 Orphan Drug Act (ODA) as well as subsequent legislation for medical devices. This article describes how OOPD assists in the development of orphan products (drugs, biologics, medical devices, and medical foods) for patients with rare diseases.

The 40 clinicians, pharmacists, scientists, engineers, and others who perform OOPD's mission activities are broadly committed to providing useful new therapies to people with rare diseases. However, OOPD does not actually decide what products (drugs, biologics devices, and medical foods) are licensed for marketing and which applications are denied. Rather, OOPD has three key functions: certifying that indeed a product has bona fide promise for use in a rare disease, granting funds for the development of such products and advocating with stakeholders for the creation of new therapies in rare disease therapies.

\section{Certifying Product Applicability to a Rare Disease}

In order to grant products for rare diseases with special consideration or financial incentives, systems are needed to

\section{E. Chen $(\bowtie)$}

Humanitarian Use Devices Designation Program, Office of Orphan Products Development, Food and Drug Administration, Silver Spring, MD, USA

e-mail: eric.chen@fda.hhs.gov certify the eligibility of particular products based on their application for a rare disease. Such a system was established by the ODA, which stipulated that a drug or biologic can be designated with orphan status if it can demonstrate

- that there are data that (liberally interpreted) support its "promise" for the treatment of a rare disease and

- that the disease occurs among fewer than 200,000 people in the USA.

Though designated with orphan status, a drug or biologic cannot yet be sold to patients - it needs to be deemed safe and efficacious by the FDA review divisions in accordance with the same standards as required of drugs for common diseases. However, orphan drugs are eligible for tax credits, exemption from the FDA filing fee of $\$ 1.5 \mathrm{M}$, and (most importantly) upon subsequent marketing approval by a review division may receive 7 years of market exclusivity (meaning no other drug-maker will be licensed to sell the same product to treat that rare disease, an incentive superior to patent protection). The ODA has had considerable success over its 27 years: more than 2,220 drugs and biologic have received orphan status and of these 361 have progressed to full market approval.

Devices are similarly designated as potentially useful in the treatment of rare diseases under the auspices of Safe Medical Devices Act of 1990 (amended in 1997 and 2007) which authorized the Humanitarian Device Exemption (HDE) pathway. To be eligible for an HDE, a manufacturer must first request that the device be designated by OOPD as a Humanitarian Use Device (HUD). In contrast to the ODA (which sets out specific financial attractions), the HUD/ HDE pathway offers an alternative means of getting to market. Standard device licensing requirements of the Premarket Approval pathway (i.e., demonstrated safety and effectiveness) or the $510(\mathrm{k})$ pathway (i.e., substantial 
equivalence), are bypassed and marketing under HDE can proceed if the product is shown to be safe and have "probable benefit". Other differences between the HUD/ HDE and the ODA include a difference in number of people with the disease (fewer than 4,000 new cases per year under HUD/HDE) and the HUD/HDE's prohibition on profit generation except for pediatric devices. The HUD/ HDE legislation has had success-150 HUDs have been designated for the needs of small populations; of these, 50 have emerged through the HDE pathway as marketed devices.

\section{Funding for Products for Rare Diseases}

FDA does not have the recognition as a grants-making institution so central to our sister agency, the National Institutes of Health (NIH); however FDA's largest grants portfolio funds the development of products for rare diseases. The Orphan Products Grants Program funds clinical trials for drugs, biologics, medical devices, and medical foods potentially useful in treating rare diseases or conditions. The objective of these grants is to develop data that can support marketing applications, and since 1983 more than 500 funded studies have yielded data that have brought 45 products to market. These grants are only for clinical trials (phase I, II, or III) and are for up to $\$ 400,000$ / year for up to 4 years. Currently, the Orphan Products Grants Program funds 60 to 85 ongoing projects and awards approximately 15 to 20 new grants per fiscal year. Approximately $\$ 15$ million are annually appropriated for this program.

The Pediatric Device Consortia Grant Program was established by Congress in 2007 based on recognition of special device needs of children. It funds nonprofit consortia that create new pediatric medical devices by linking innovators and manufacturers. The consortia mentor and manage pediatric device projects, offering regulatory and business advice. At this writing, $\$ 3 \mathrm{M}$ is allocated annually to four consortia: the Michigan Pediatric Device Consortium, The Pediatric Cardiovascular Device Consortium, the University of California, San Francisco Pediatric
Device Consortium, and the MISTRAL Device Consortium. Approximately 50 pediatric medical devices have received assistance by the consortia.

\section{Advocating for New Therapies for Rare Diseases}

While not engaged in direct product licensing, OOPD serves as the agency's focal point for therapies for rare diseases, an endeavor that blends the efforts of manufacturers, patient advocacy groups, academia, clinicians, and international bodies. OOPD coordinates FDA's productive outreach to these stakeholders, and conducts "in-reach" to other FDA staff to sensitize the agency to the special challenges of developing products for people with rare diseases. Accordingly, OOPD has been charged with such disparate activities as collecting public comments through Part 15 meetings, collaborating with European Medicines Agency on international orphan drug regulation, sponsoring a course on alternative methodologies in small clinical trials and preparing a forthcoming report to Congress that addresses FDA's broad approach to articles used in the treatment of rare diseases. These outreach/inreach activities are enhanced by the analytical team by their utilization of the considerable data on therapeutics development accumulated over 27 years of operation.

\section{Conclusion}

FDA regulates the use of articles for the treatment of diseases, but treatments for rare diseases have special challenges that require special efforts. Not only do they require dispassionate consideration of data on product safety and efficacy, but also they require systems that certify their promise for a rare disease, funding for clinical trials/device consortia and coordinated outreach to manufacturers, patients, academics, and others. While not directly engaged in the marketing approval review process, OOPD has served prominent roles in advancing remarkable new therapies for people with rare diseases. 